\title{
Analyzing Infant Mortality in Rural Bangladesh: A Frailty Modeling Approach
}

\author{
Bikash Pal and Ahsan Rahman Jaamee \\ Department of Statistics, Dhaka University, Dhaka-1000, Dhaka, Bangladesh
}

(Received : 31 August 2020; Accepted : 14 October 2021)

\begin{abstract}
In practice, it may happen that data may arise from a hierarchical structure i.e., a cluster is nested within another cluster. In this case, nested frailty model is appropriate to analyze survival data to obtain optimal estimates of the parameters of interest. To identify significant determinants of infant mortality in rural Bangladesh, survival data have been extracted from Bangladesh Demographic and Health Survey (BDHS), 2014. Because of the presence of two-level clustering in data, nested frailty model has been employed for the purpose of analysis. Recommendations have been suggested based on the results obtained from the survival model to reduce the infant mortality in rural Bangladesh to a great extent.
\end{abstract}

Keywords: hierarchical structure, nested frailty model, survival data, infant mortality

\section{Introduction}

Mortality of children is one of the main indicators of socioeconomic development of a country ${ }^{1,2}$. This is also a crucial problem in developing countries of the world. Though Bangladesh has made a significant progress to reduce child mortality over the past few years, the rate still remains high $^{3}$. In Bangladesh, several programs have been taken to reduce urban-rural and regional inequalities in child mortality but socioeconomic inequalities still continuing ${ }^{4}$. Infant mortality generally depends on environmental, social, and cultural factors in developing countries. As a result, it differs substantially between rural and urban areas. Moreover, economic disadvantage, poor transportation systems, and religious conservatism contribute much to infant mortality in rural Bangladesh ${ }^{5}$. In addition, socioeconomic, environmental, ethnical and genetic factors create clustering effect, which in terms influence infant mortality ${ }^{6}$. The event of infant mortality from infectious and diarrheal diseases is less in urban areas for better health care facilities and water supply. Urban guardians provide better environment for infants than the rural guardians. In contrast, high risk of infant death exists in rural areas because of the lacking of pure drinking water, sanitary and healthcare facilities, high illiteracy, and a high incidence of poverty ${ }^{5}$.

Almost all the large surveys in the developing countries collect clustered data in which the clusters are the geographic areas. As the individuals in the same cluster have some common factors in them, the corresponding survival times tend to be correlated ${ }^{7}$. For instance, if the data come from the same community or same households, then the observations must have some association among themselves. This association is created due to the unobservable or unmeasurable environmental, genetic, and behavioral factors that are related to the event of interest. In the same way, heterogeneity exists among the observations from different clusters. Frailty model considers the sharing of these unobserved factors of the observations of the same cluster as well as the heterogeneity of the observations from different clusters for estimating the parameters of interest ${ }^{8}$. Therefore, ignoring these heterogeneity and dependency under clustered sampling scheme may provide incorrect standard errors of the estimates of the parameters ${ }^{9}$. Frailty models cover this unobserved variation through the frailty term, which is a non-negative valued random variable. Gamma, inverse Gaussian, log-normal, positive stable, etc. are used as the distribution of the frailty term. Mathematical tractability of Gamma frailty model has made it widely used frailty model ${ }^{10}$.

It may happen, in practice that data are collected using twostage cluster sampling, where, for example, the first level is community and the second level is household. Under this setup, observations within households are correlated and households within community are also correlated ${ }^{11}$. To obtain the optimal estimates of parameters of interest, it is required to consider both correlation into account. To address such situation, in survival analysis, the nested frailty model has widely been used ${ }^{12}$. In this paper, the mortality of children aged below one year in the rural Bangladesh has been considered as the event of interest. For the purpose of analysis, a sample from Bangladesh Demographic and Health Survey (BDHS), 2014 kids record data has been extracted and analyzed using nested frailty model.

In this paper, nested frailty model has been considered to assess the impact of significant covariates on infant mortality of children in rural Bangladesh and recommending suggestions following results from the fitted model. In the following sections, Methods, a short description of nested frailty model along with the inference procedure and overview of data and variables are given. Then the obtained results from univariate, bivariate analysis, and nested frailty model have been presented. In the penultimate section, a brief discussion of the obtained results is provided and finally the paper concludes.

\section{Methods}

A semi-parametric modeling approach has been used in this study to measure the effect of covariates on the lifetime $T$. Cox (1972) proposed a semi-parametric proportional hazard model, which takes the form as ${ }^{13}$ 


$$
h(t \mid \boldsymbol{x})=h_{0}(t) \exp \left(\boldsymbol{\beta}^{\prime} \boldsymbol{x}\right),
$$

where $h_{0}(t)$ be the baseline hazard function and $\boldsymbol{x}$ be the vector of covariates associated with the vector of regression parameters $\boldsymbol{\beta}$.

Nested frailty model is used for capturing the hierarchical clustering effect of the data by including two random effect terms that work multiplicatively on the hazard function. These models are appropriate when data are clustered at hierarchical levels naturally or by design ${ }^{14}$. Let's consider $g$ independent clusters and within the $i$ th cluster there are $n_{i}$ subclusters. Let $T_{i j k}$ denotes the survival time under study for the $k$ th subject $\left(k=1,2, \ldots, d_{i j}\right)$ from the $j$ th subcluster $\left(j=1,2, \ldots, n_{i}\right)$ of the $i$ th cluster $(i=1,2, \ldots, g)$. Then the hazard function for $k$ th individual in nested frailty model is given by

$$
\begin{gathered}
h_{i j k}\left(t \mid u_{i} u_{i j}\right)=h_{0}(t) u_{i} u_{i j} \exp \left(\boldsymbol{\beta}^{\prime} \boldsymbol{x}\right), \\
i=1,2, \ldots, g, j=1,2, \ldots, n_{i}
\end{gathered}
$$

where the cluster random effect $u_{i}$ and the sub-cluster random effect $u_{i j}$ are both independently and identically distributed having nonnegative valued distributions. The mean of the distributions for both $u_{i}$ and $u_{i j}$ are constrained to one and the unknown variances are $\alpha$ and $\eta$, respectively. It is very important to choose frailty distribution properly. Any nonnegative random variable can be used to model frailty ${ }^{15}$. For mathematical simplicity, Gamma distribution has been used in this study as the frailty distribution.

\section{Parameter Estimation}

Let $T_{1}, T_{2}, \ldots, T_{l}$ be the observed distinct ordered times at which events occurred. Also, let $R_{t}$ be the risk set at time $T_{t}$, which consists of the set of individuals who are available in the study at time $T_{t}$. Suppose $l$ distinct observed times are found in a censored random sample of size $n$ and $d_{t}$ be the number of events occurred at time $T_{t}$. In the case of tie, the overall likelihood function for the vector of parameters $\boldsymbol{\theta}$ can be written as

$$
\begin{gathered}
L(\boldsymbol{\theta})=\prod_{i=1}^{g}\left[\int _ { 0 } ^ { \infty } \left(\prod_{j=1}^{n_{i}}[\right.\right. \\
\int_{0}^{\infty} \prod_{t=1}^{l_{i j}} \frac{\prod_{k \epsilon D_{t}} \exp \left(\boldsymbol{\beta}^{\prime} \boldsymbol{x}_{\boldsymbol{k}}\right) u_{i} u_{i j}}{\left(\prod_{k \in R_{t}} \exp \left(\boldsymbol{\beta}^{\prime} \boldsymbol{x}_{\boldsymbol{k}}\right) u_{i} u_{i j}\right)^{D_{t}}} \\
\left.\left.\left.\frac{u_{i}^{\frac{1}{\alpha}-1} \exp \left(-\frac{u_{i}}{\alpha}\right)}{\alpha^{\frac{1}{\alpha}} \Gamma\left(\frac{1}{\alpha}\right)} d u_{i j}\right] \frac{u_{i}^{\frac{1}{\eta}-1} \exp \left(-\frac{u_{i}}{\eta}\right)}{\eta^{\frac{1}{\eta}} \Gamma\left(\frac{1}{\eta}\right)}\right) d u_{i}\right] .
\end{gathered}
$$

To obtain optimal estimates of the parameters, the penalized log-likelihood function of the above likelihood function has been optimized by the robust Marquardt algorithm $^{16}$, which is a combination between a Newton Raphson algorithm and a steepest descent algorithm under the function "frailtyPenal" of R package "frailtypack".

\section{Data and Variables}

The data set used in this study have been extracted from Bangladesh Demographic and Health Survey (BDHS), 2014. Two-stage stratified sample of households is used in conducting the survey. In the first stage, 600 EAs are selected with probability proportional to the EA size and in the second-stage of sampling, on an average, a systematic sample of 30 households has been selected per EA to provide statistically reliable estimates for key demographic and health related variables for the country as a whole, for both urban and rural areas separately. The event of interest is infant mortality in the rural Bangladesh, and the corresponding independent variables, based on previous literature review, are region [Dhaka, Barisal, Chittagong, Khulna, Rajshahi, Rangpur, Sylhet], maternal education [No, Primary, Secondary, Higher], source of drinking water [Safe and Unsafe], toilet facilities [Safe and Unsafe], domestic violence [Yes or No], awareness about child health care [Respondent and Others], NGO membership [Non-member, Member], access to media [Unexposed and Exposed], working status of mother [Yes or No], age of mother at first birth [Less than or equal to 20 year and Above 20 year], sex of child [Male and Female], birth order number [Other than first birth and First birth], religion [Muslim and Non-Muslim], preceding birth interval [Less than equal to 18 months and Above 18 months], fathers education [No, Primary, Secondary, Higher], sex of household head [Male, Female], number of antenatal care visit [Less than 4, More than or equal to 4], and fathers occupation [Farmer, Service or business, Labor or others]. All these variables have been categorized according to previous literature review, and after deleting missing cases, 4889 observations have been found ready for analysis.

\section{Results}

\section{Univariate Analysis}

The rural respondents are more or less equally distributed among the divisions. Chittagong division has the highest percentage $(18.8 \%)$ of respondents and Khulna division has the lowest one $(10.7 \%)$. Though very few $(6.4 \%)$ respondents are highly educated, almost half of them have completed secondary education $(45.5 \%)$, while about onethird have primary education $(30.1 \%)$. But, still $18 \%$ respondents having no education is a matter of concern. It's a matter of joy that $96.6 \%$ and $91.5 \%$ of the respondents have safe drinking water and toilet facility, respectively. Exactly one-third (33.3\%) respondents are victim of domestic violence. $69.2 \%$ respondents have taken health care of their child by themselves. It has been found that $30.8 \%$ mothers have not taken direct care of their children, which is not a small percentage indeed. Only $32.7 \%$ respondents are NGO members. Almost half (49.3\%) of the respondents are exposed to media implying still $50.7 \%$ have had no opportunity to be exposed. Most of the respondents $(85 \%)$ have had their first birth at or before age 20 , which is obviously a big issue to concern. There are almost equal percentage $(51.4 \%$ and $48.6 \%$ ) of male and female child, 
respectively, in this study. Only $34.9 \%$ of the children are first birth to their mothers. Majority $(72.8 \%)$ of the respondents are non-worker. Among the respondents, 92.1\% are Muslim. 79.3\% respondents have at least 18month gap between taking index birth and previous birth. It should be noted that for the first birth, time to take first baby after marriage is considered as the preceding birth interval. Most $(91 \%)$ of the household heads are male. Very few $(9.7 \%)$ fathers have higher education, whereas almost one-third $(29.3 \%)$ have no education, one-third $(33 \%)$ have primary, and rest one-third (28\%) have secondary education. Almost equal percentage of fathers $(29.6 \%$ and $27.8 \%$ ) are farmer and service/businessmen, respectively. Rest of them $(42.2 \%)$ are labor or in other professions. In our study, most of the rural mothers $(74.5 \%)$ took antenatal care visit less than four times.

\section{Bivariate Analysis}

The bivariate technique involving the Product-Limit estimator $^{17}$ for survival function along with log-rank test ${ }^{18}$ has been used, rather than other ways of bivariate analysis like correlation, cross-tabulation, measure of association by chi-square test, as these techniques can't handle censoring properly. Table 1 presents the comparison of the survival probabilities for socio-economic and demographic variables along with log-rank test $\mathrm{p}$-value. The variables with significant impact on mortality of infants in rural Bangladesh at $5 \%$ level of significance are Region, Toilet Facility, Domestic Violence, Access to Media, Preceding Birth Interval, and Sex of Household Head; whereas Maternal education, Source of Drinking Water, Awareness about Child Health Care, NGO Membership, Age of Mother at First Birth, Sex of Child, Birth Order Number, Working Status of Mother, Religion, Fathers Education, Number of Antenatal Care Visit, and Fathers occupation are found to have no significant association. Along with the bivariate significant variables, NGO Membership, Religion, Working Status of Mother and Birth Order Number are also included in the nested frailty model as the long rank test pvalues corresponding to these covariates are small.

Table 1. Log-rank test of survival times between the different categories for the selected socio-economic and demographic variables on infant mortality in rural Bangladesh from BDHS, 2014 data

\begin{tabular}{|c|c|c|}
\hline Covariates & $\begin{array}{l}\text { Survival Probability (at } \\
\text { most of the time points) }\end{array}$ & p-value \\
\hline \multicolumn{2}{|l|}{ Region } & 0.019 \\
\hline Dhaka & Barisal $>$ Dhaka $>$ Chittagong $>$ & \\
\hline Barisal & Rajshahi $>$ Rangpur $>$ Khulna $>$ & \\
\hline Chittagong & Sylhet & \\
\hline Khulna & & \\
\hline Rajshahi & & \\
\hline Rangpur & & \\
\hline Sylhet & & \\
\hline \multicolumn{2}{|l|}{ Maternal Education } & 0.280 \\
\hline No education & Higher $>$ Secondary $>$ Primary $>$ & \\
\hline Primary & No education & \\
\hline Secondary & & \\
\hline Higher & & \\
\hline \multicolumn{2}{|l|}{ Source of Drinking Water } & 0.580 \\
\hline Safe & Safe $>$ Unsafe & \\
\hline \multicolumn{3}{|l|}{ Unsafe } \\
\hline Toilet Facility & & 0.006 \\
\hline Safe & Safe $>$ Unsafe & \\
\hline Unsafe & & \\
\hline \multicolumn{2}{|l|}{ Domestic Violence } & 0.004 \\
\hline No & No $>$ Yes & \\
\hline Yes & & \\
\hline \multicolumn{2}{|l|}{ Awareness about Child Health Care } & 0.240 \\
\hline Respondent & Respondent $>$ Others & \\
\hline Others & & \\
\hline \multicolumn{2}{|l|}{ NGO Membership } & 0.076 \\
\hline Non-member & Non-member $>$ Member & \\
\hline Member & & \\
\hline \multicolumn{2}{|l|}{ Access to Media } & 0.044 \\
\hline Unexposed & Exposed $>$ Unexposed & \\
\hline Exposed & & \\
\hline \multicolumn{2}{|l|}{ Age of Mother at First Birth } & 0.850 \\
\hline Less than or equal to 20 years & Less than or equal to 20 years $>$ & \\
\hline Above 20 years & Above 20 years & \\
\hline
\end{tabular}




\begin{tabular}{|c|c|c|}
\hline Covariates & $\begin{array}{l}\text { Survival Probability (at } \\
\text { most of the time points) }\end{array}$ & p-value \\
\hline Sex of Child & & 0.950 \\
\hline Female & Male $>$ Female & \\
\hline \multicolumn{3}{|l|}{ Male } \\
\hline Birth Order Number & & 0.150 \\
\hline Other than first birth & Other than first birth $>$ First birth & \\
\hline \multicolumn{3}{|l|}{ First birth } \\
\hline Working Status of Mother & & 0.140 \\
\hline No & No $>$ Yes & \\
\hline \multicolumn{3}{|l|}{ Yes } \\
\hline Religion & & 0.085 \\
\hline Non-Muslim & Muslim > Non-Muslim & \\
\hline \multicolumn{3}{|l|}{ Muslim } \\
\hline Preceding Birth Interval & & 0.021 \\
\hline Less than or equal to 18 months & Above 18 months $>$ & \\
\hline Above 18 months & Less than or equal to 18 months & \\
\hline Fathers Education & & 0.100 \\
\hline No education & Higher $>$ Secondary $>$ Primary $>$ & \\
\hline Primary & No education & \\
\hline \multicolumn{3}{|l|}{ Secondary } \\
\hline \multicolumn{3}{|l|}{ Higher } \\
\hline Sex of Household Head & & 0.030 \\
\hline Male & Female $>$ Male & \\
\hline \multicolumn{3}{|l|}{ Female } \\
\hline Number of Antenatal Care Visit & & 0.800 \\
\hline Less than 4 & More than or equal to $4>$ & \\
\hline More than or equal to 4 & Less than 4 & \\
\hline Fathers Occupation & & 0.500 \\
\hline Farmer & Service or business $>$ & \\
\hline Service or business & Labor or others $>$ Farmer & \\
\hline Labor or others & & \\
\hline
\end{tabular}

\section{Multivariable Analysis}

Nested frailty model has been employed to analyze infant mortality data in rural Bangladesh with the selected variables from bivariate analysis and the results are shown in Table 2. It is found that the variables Region, Toilet Facility, Domestic Violence, NGO Membership, Working Status of Mother, Birth Order Number, Religion, and Sex of Household Head have significant impact on infant mortality, whereas Access to Media and Preceding Birth Interval is insignificant. The estimated frailty variance terms for both the community and mother level are significant with p-values less than 0.001 and 0.026 , respectively. This clearly indicates the presence of dependency in infant mortality data for both community and mother level.

It is found from Table 2 that the hazard of occurring infant mortality for a child residing in rural Barisal division is $(1-0.812) \times 100 \%=18.8 \%$ lower than that of a child from rural Dhaka division. Similarly, for a child from rural Chittagong (43.9\%), Khulna (79.3\%), Rajshahi (9.5\%), Rangpur (29.6\%), and Sylhet division (152.4\%) have higher hazard of having infant mortality than that of a child from rural Dhaka division. Here, the hazard ratio of a child from rural Sylhet division varies significantly from that of Dhaka division with p-value 0.003 . The hazard of occurring infant death for a rural child who belongs to the household with unsafe toilet facilities is 1.725 times compared to that of a child from household with safe toilet facilities with $\mathrm{p}$-value 0.051. Domestic violence has been turned out as a potential determinant of infant mortality in rural Bangladesh ( $p$-value $=0.037$ ). If a mother is victim to domestic violence, her child has 1.449 times as much as hazard of occurring infant mortality compared to a child who is from non-victimized mother. Moreover, a rural child whose mother is member of NGO has 1.540 times as much as hazard of dying before celebrating first birthday compared to a child from nonmember mother and this difference is significant with $\mathrm{p}$-value 0.022. In this study, for the variable Birth Order Number, a child who is first birth to his/her mother has $92.4 \%$ higher hazard of occurring infant mortality than that of a child who is other than first birth to his/her mother with p-value 0.010 . It has been found that the hazard of occurring infant mortality for a rural child whose mother works outside is significantly ( $\mathrm{p}$-value $=0.015)$ 63.7\% higher than that of a child whose mother doesn't work outside. In this study, religion is also a potential risk factor for infant mortality ( $\mathrm{p}$-value $=0.005)$. A child from rural Muslim mother has 2.993 times as much as hazard of occurring infant mortality compared to a child who is from non-Muslim mother. It is also evident that the rural child who belongs to the family headed by female has significantly $50.9 \%$ lower hazard of occurring infant mortality than the child whose family is headed by male with p-value 0.054 . 
Table 2. Nested frailty model estimates of the selected variables for infant mortality in rural Bangladesh from BDHS, 2014 data along with standard error (SE), hazard ratio (HR) and p-value

\begin{tabular}{|c|c|c|c|c|}
\hline Covariates & Coefficient & SE & HR & p-value \\
\hline \multicolumn{5}{|l|}{ Region } \\
\hline Dhaka & - & - & - & - \\
\hline Barisal & -0.207 & 0.382 & 0.812 & 0.587 \\
\hline Chittagong & 0.364 & 0.323 & 1.439 & 0.262 \\
\hline Khulna & 0.584 & 0.348 & 1.793 & 0.093 \\
\hline Rajshahi & 0.090 & 0.349 & 1.095 & 0.794 \\
\hline Rangpur & 0.260 & 0.343 & 1.296 & 0.447 \\
\hline Sylhet & 0.925 & 0.318 & 2.524 & 0.003 \\
\hline \multicolumn{5}{|l|}{ Toilet Facility } \\
\hline Safe & - & - & - & - \\
\hline Unsafe & 0.545 & 0.280 & 1.725 & 0.051 \\
\hline \multicolumn{5}{|l|}{ Domestic Violence } \\
\hline No & - & - & - & - \\
\hline Yes & 0.371 & 0.178 & 1.449 & 0.037 \\
\hline \multicolumn{5}{|l|}{ NGO Membership } \\
\hline Non-member & - & - & - & - \\
\hline Member & 0.432 & 0.189 & 1.540 & 0.022 \\
\hline \multicolumn{5}{|l|}{ Access to Media } \\
\hline Unexposed & - & - & - & - \\
\hline Exposed & -0.293 & 0.183 & 0.745 & 0.109 \\
\hline \multicolumn{5}{|l|}{ Birth Order Number } \\
\hline Other than first birth & - & - & - & - \\
\hline First birth & 0.654 & 0.254 & 1.924 & 0.010 \\
\hline \multicolumn{5}{|l|}{ Working Status of Mother } \\
\hline No & - & - & - & - \\
\hline Yes & 0.493 & 0.202 & 1.637 & 0.015 \\
\hline \multicolumn{5}{|l|}{ Religion } \\
\hline Non-Muslim & - & - & - & - \\
\hline Muslim & 1.096 & 0.393 & 2.993 & 0.005 \\
\hline \multicolumn{5}{|l|}{ Preceding Birth Interval } \\
\hline Less than or equal to 18 months & - & - & - & - \\
\hline Above 18 months & -0.019 & 0.230 & 0.980 & 0.933 \\
\hline \multicolumn{5}{|l|}{ Sex of Household Head } \\
\hline \multicolumn{5}{|l|}{ Male } \\
\hline Female & -0.709 & 0.368 & 0.491 & 0.054 \\
\hline \multicolumn{5}{|l|}{ Variance of Random Effects } \\
\hline Community & 0.007 & $<0.001$ & & $<0.001$ \\
\hline Mother & 6.070 & 3.141 & & 0.026 \\
\hline Log-likelihood & -1252.78 & & & \\
\hline
\end{tabular}

\section{Discussion}

The main focus of this work is to search for the potential determinants on infant mortality in rural Bangladesh through analyzing the data obtained from Bangladesh Demographic and Health Survey (BDHS), 2014. Because of the presence of two-stage clustering effect in the data, nested frailty model has been used to find optimal results. The analysis reveals that there exists significant regional difference, which should be reduced to decrease the rural infant mortality. Similar result has been found in previous study conducted in Bangladesh ${ }^{4}$. Survival of children before 
reaching first year in life is significantly worse for the rural children of Sylhet division of Bangladesh than those residing in the rural Dhaka division. This high risk may occur due to the territorial advancement, social improvement activity in the division, population density, as well as regional economic resources ${ }^{7}$. Toilet facility has been found to have a significant impact for infant death, which is due to the fact that unsafe toilet system causes many virus-bacteria related diseases. These diseases are a great threat to the lives of the children younger than one year ${ }^{19}$. Children residing in the households with safe toilet facilities get a healthy environment in their childhood and this is why, they immune to several diseases ${ }^{1}$. This study also finds the evidence that domestic violence causes more death to the infants. The victim mothers may not take proper care of their children due to this violence ${ }^{20}$. The children from NGO member mothers have the lowest survival probability, which may happen because the NGO members are usually too poor to have education and other facilities and also the rich NGO members are too busy to take care of their children ${ }^{21}$. The analysis also suggests that infant mortality is worse for the children who are first birth to their mothers compared to the children who are other than first birth to their mothers. The cause might be that first birth babies are coming from younger mothers and these mothers may face complicacy during the period of pregnancy and at the delivery time because of the physical immaturity $^{22}$. In addition, these mothers may have limited knowledge about their child health ${ }^{23}$. Similar result has been found on child mortality that infant death is higher for the children from working mother ${ }^{6,24}$. It is easy to understand that working mothers can't manage enough time for their children. Even, in many cases, maternity leave is also not available according to the demand, which is responsible for causing harm to the upcoming baby ${ }^{24}$. Religion has been found significant in this study. The children from Muslim mothers are more prone to die before celebrating their first birthday ${ }^{25}$. The rural Muslim families may lack in proper health and education facilities to bring up their babies carefully ${ }^{26}$. It is also important for infant mortality that whether the household head is male or female ${ }^{27}$. Our study finds that if the head of the household is a woman, there prevails lower chance of infant mortality.

\section{Conclusion}

A dataset has been extracted from Bangladesh Demographic and Health Survey (BDHS), 2014 kids record file and analyzed using nested frailty model to find out the determinants that have potential impact on infant mortality in rural Bangladesh. The obtained result suggests that rural mothers of Sylhet division should be given extra attention to enhance awareness programs for reducing infant mortality gradually. The use of hygienic toilets should be enhanced among the rural families. Domestic violence should be stopped to reduce infant mortality as it has been found significant in this study. NGOs should pay more attention to the pregnant members by lessening their working pressure and providing valuable information on child health care. The rural working mothers should become more careful about their maternity. Also, more awareness is needed for the Muslim mothers on their maternity period. As the first baby of the parents are more prone to death, information on pregnancy complication and child health care should be provided to the newly married mothers.

\section{References}

1. Mondal MNI, MK Hossain, MK Ali, 2009. Factors influencing infant and child mortality: A case study of Rajshahi District, Bangladesh. Journal of Human Ecology, 26(1), 31-9.

2. Vijay, J., Patel, K. K., 2020. Risk factors of infant mortality in Bangladesh. Clinical Epidemiology and Global Health, 8(1), 211-214.

3. Mohammad KA, W Bari, 2017. Fine and Gray competing risk regression model to study the cause-specific under-five child mortality in Bangladesh. BMC International Health and Human Rights, 17(1), 3.

4. Abir T, KE Agho, AN Page, AH Milton, MJ Dibley, 2015. Risk factors for under-5 mortality: evidence from Bangladesh Demographic and Health Survey, 2004-2011. BMJ Open, 5(8), e006722.

5. Paul BK, 1990. Factors Affecting Infant Mortality in Rural Bangladesh: Results from a Retrospective Sample Survey 1. Rural Sociology, 55(4), 522-40.

6. Gruebner O, M Khan, K Burkart, S Lautenbach, T Lakes, A Krämer, et al, 2017. Spatial variations and determinants of infant and under-five mortality in Bangladesh. Health \& Place, 47, 156-64.

7. Khan JR, N Awan, 2017. A comprehensive analysis on child mortality and its determinants in Bangladesh using frailty models. Archives of Public Health, 75(1), 58.

8. Sastry N, 1997. Family-level clustering of childhood mortality risk in Northeast Brazil. Population Studies, 51(3), 245-61.

9. Mahmood S, B Zainab, AM Latif, 2013. Frailty modeling for clustered survival data: an application to birth interval in Bangladesh. Journal of Applied Statistics, 40(12), 2670-80.

10. Munda M, F Rotolo, C Legrand, 2012. Parfm: parametric frailty models in R. Journal of Statistical Software, 51(11), 120.

11. Khan, H. R., Shaw, E., 2011. Multilevel logistic regression analysis applied to binary contraceptive prevalence data. Journal of Data Science, 9, 93-110.

12. Kim S, DK Dey, 2008. Modeling multilevel survival data using frailty models. Communications in Statistics-Theory and Methods, 37(11), 1734-41.

13. Cox DR, 1972. Regression models and life-tables. Journal of the Royal Statistical Society: Series B (Methodological), 34(2), 187-202.

14. Rondeau V, Y Mazroui, JR Gonzalez, 2012. frailtypack: an R package for the analysis of correlated survival data with frailty models using penalized likelihood estimation or parametrical estimation. Journal of Statistical Software, 47(4), 1-28. 
15. Vaupel JW, KG Manton, E Stallard, 1979. The impact of heterogeneity in individual frailty on the dynamics of mortality. Demography, 16(3), 439-54.

16. Marquardt, DW, 1963. An algorithm for least-squares estimation of nonlinear parameters. Journal of the Society for Industrial and Applied Mathematics, 11(2), 431-441.

17. Kaplan EL, P Meier, 1958. Nonparametric estimation from incomplete observations. Journal of the American Statistical Association, 53(282), 457-81.

18. David G, K Kleinbaum, 2016. Survival analysis: a selflearning text: Springer-Verlag New York.

19. Majumder AK, SS Islam, 1993. Socioeconomic and environmental determinants of child survival in Bangladesh. Journal of Biosocial Science, 25(3), 311-8.

20. Åsling-Monemi K, R Tabassum Naved, LÅ Persson, 2008. Violence against women and the risk of under-five mortality: analysis of community-based data from rural Bangladesh. Acta Paediatrica, 97(2), 226-32.

21. Fatima-Tuz-Zahura M, KA Mohammad, W Bari, 2017. LogLogistic Proportional Odds Model for Analyzing Infant
Mortality in Bangladesh. Asia Pacific Journal of Public Health, 29(1), 60-9.

22. Alam N, 2000. Teenage motherhood and infant mortality in Bangladesh: maternal age-dependent effect of parity one. Journal of Biosocial Science, 32(2), 229-36.

23. Swenson I, 1981. Relationships between pregnancy spacing, sex of infants, maternal age, and birth order, and neonatal and post-neonatal mortality in Bangladesh. Social Biology, 28(34), 299-307.

24. Ahmad-Nia S, 2002. Women's work and health in Iran: a comparison of working and non-working mothers. Social Science \& Medicine, 54(5), 753-65.

25. Zenger E, 1993. Siblings' neonatal mortality risks and birth spacing in Bangladesh. Demography, 30(3), 477-88.

26. Hossain M, M Islam, 2009. Socio-economic variables affecting infants and children mortality in Bangladesh. Internet J Health, 9(2).

27. Gupta, A. K., Borkotoky, M. K., Kumar, A., 2015. Household headship and infant mortality in India: evaluating the determinants and differentials. International Journal of $M C H$ and AIDS, 3(1), 44. 